\title{
Active animal health surveillance in European Union Member States: gaps and opportunities
}

B. BISDORFF ${ }^{1} \dagger$, B. SCHAUER ${ }^{2,3 * \dagger, ~ N . ~ T A Y L O R ~}{ }^{1,4}$, V. RODRÍGUEZ-PRIETO ${ }^{5}$, A. COMIN ${ }^{6}$, A. BROUWER ${ }^{7}$, F. DÓREA ${ }^{6}$, J. DREWE $^{1}$, L. HOINVILLE $^{1}$, A. LINDBERG ${ }^{6}$, M. MARTINEZ AVILÉS ${ }^{5}$, B. MARTÍNEZ-LÓPEZ ${ }^{5,8}$, M. PEYRE ${ }^{9}$, J. PINTO FERREIRA ${ }^{10}$, J. RUSHTON $^{1}$, G. VAN SCHAIK ${ }^{11}$, K. D. C. STÄRK ${ }^{10}$, C. STAUBACH ${ }^{2}$, M. VICENTE-RUBIANO ${ }^{5}$, G. WITTEVEEN ${ }^{11}$, D. PFEIFFER ${ }^{1}$ AND B. HÄSLER ${ }^{1,12}$

${ }^{1}$ Veterinary Epidemiology, Economics and Public Health Group, Royal Veterinary College (RVC), Hatfield, North Mymms, UK; ${ }^{2}$ Institute of Epidemiology, Friedrich-Loeffler-Institut (FLI), Greifswald-Insel Riems, Germany; ${ }^{3}$ Institute for Community Medicine, University Medicine Greifswald (UMG), Greifswald, Germany;

${ }^{4}$ Veterinary Epidemiology and Economics Research Unit, University of Reading, Reading, UK;

${ }^{5}$ VISAVET Centre, Veterinary faculty, Complutense University (UCM), Madrid, Spain; ${ }^{6}$ Swedish Zoonosis Centre Department of Disease Control and Epidemiology, National Veterinary Institute (SVA), Uppsala, Sweden; ${ }^{7}$ Department of Epidemiological Sciences, Animal and Plant Health Agency (APHA), Weybridge, UK;

${ }^{8}$ Center for Animal Disease Modeling and Surveillance, UC Davis, CA, USA; ${ }^{9}$ AGIRs-Animal and Integrated Risk Management Research Unit, CIRAD-French Agricultural Research Center for International Development, Montpellier, France; ${ }^{10}$ SAFOSO AG, Liebefeld, Switzerland; ${ }^{11}$ Department of Research and Development, GD Animal Health, Deventer, The Netherlands; ${ }^{12}$ Leverhulme Centre for Integrative Research on Agriculture and Health, Royal Veterinary College, Hatfield, North Mymms, UK

Received 26 July 2016; Final revision 19 October 2016; Accepted 26 October 2016; first published online 12 December 2016

\section{SUMMARY}

Animal health surveillance enables the detection and control of animal diseases including zoonoses. Under the EU-FP7 project RISKSUR, a survey was conducted in 11 EU Member States and Switzerland to describe active surveillance components in 2011 managed by the public or private sector and identify gaps and opportunities. Information was collected about hazard, target population, geographical focus, legal obligation, management, surveillance design, risk-based sampling, and multi-hazard surveillance. Two countries were excluded due to incompleteness of data. Most of the 664 components targeted cattle $(26 \cdot 7 \%)$, pigs $(17 \cdot 5 \%)$ or poultry $(16 \cdot 0 \%)$. The most common surveillance objectives were demonstrating freedom from disease $(43.8 \%)$ and case detection (26.8\%). Over half of components applied risk-based sampling $(57 \cdot 1 \%)$, but mainly focused on a single population stratum (targeted risk-based) rather than differentiating between risk levels of different strata (stratified risk-based). About a third of components were multi-hazard $(37 \cdot 3 \%)$. Both risk-based sampling and multi-hazard surveillance were used more frequently in privately funded components. The study identified several gaps (e.g. lack of systematic documentation, inconsistent application of terminology) and opportunities (e.g. stratified riskbased sampling). The greater flexibility provided by the new EU Animal Health Law means that systematic evaluation of surveillance alternatives will be required to optimize cost-effectiveness.

Key words: EU Animal Health Law, One Health, risk-based, RISKSUR, surveillance component.

\footnotetext{
* Author for correspondence: Dr B. Schauer, Institute for Community Medicine, University Medicine Greifswald, Walther-Rathenau-Strasse 48, 17487 Greifswald, Germany. (Email: Birgit.Schauer@uni-greifswald.de)

$\dagger$ These authors contributed equally to this work.
} 


\section{INTRODUCTION}

Animal health surveillance (AHS) forms a key element in detecting and controlling animal and zoonotic diseases, demonstrating disease freedom to ensure safe trade and providing valuable data for decision support [1]. A key feature of surveillance is the need for the systematic (continuous or repeated) measurement, collection, collation, analysis, interpretation, and timely dissemination of data [2]. Hence, single surveys or analytical studies do not fall under this surveillance definition. The need for systematic analysis and interpretation arises from the close link between surveillance and intervention strategies in case undesired changes are observed [3], which distinguishes surveillance from monitoring systems. Monitoring, i.e. the collection of animal health data without a clear related action plan, usually only applies when the aim is to assess the initial health status of a population, while otherwise data collection without a clear related action plan should not be encouraged [4]. In contrast to passive surveillance, which relies on the detection and reporting of clinical signs, active surveillance is initiated by the investigator using a defined protocol to perform actions that are scheduled in advance [2]. While public health surveillance commonly relies on notifiable disease reporting (passive surveillance) and the analysis of secondary data, AHS places stronger emphasis on collecting primary data via active surveillance for example to fulfil trade requirements and ensure food safety.

In the European Union (EU) AHS is regulated by the Community Animal Health Policy (CAHP), which aims to reduce the negative impact of animal diseases on animal and public health, animal welfare, and the economy by ensuring safe intra-community trade with live animals and animal products [5]. Historically, the CAHP included almost 50 basic directives and regulations and 400 pieces of secondary legislation, most of which were adopted between 1988 and 1995 [1]. An external evaluation launched by the Commission in 2004 to assess the performance of the CAHP concluded that it lacks an overall strategy, places insufficient focus on disease prevention and does not provide enough flexibility to adapt new scientific and technological developments. Audit reports also indicated different interpretations of requirements e.g. regarding specifications of risk categories as part of the multi-annual national control plan, resulting in diverse surveillance approaches taken by Member States [6]. The new EU Animal Health Law published in March 2016 [7] is based on the EU Animal Health Strategy 'Prevention is better than cure' [5] and streamlines the huge number of legal acts into a single law, which shall also prevent piecemeal and crisisdriven policy development in the future [1]. Besides EU regulations, national and regional requirements as well as private initiatives exist, which vary between countries. Hence, the surveillance landscape in the EU includes a mixture of regulated and non-regulated activities managed by the public sector, private sector or both.

Given budget limitations, it is crucial to carefully design and regularly evaluate surveillance systems to optimize cost-effectiveness. Traditionally, input-based standards were applied, which required specific activities to be carried out regardless of the characteristics of the population. In recent years, considerable progress has been made regarding surveillance design. Alternative approaches include the application of output-based standards [8,9], where surveillance is designed to meet defined requirements (surveillance sensitivity, design prevalence), thus supporting flexible approaches targeted to the characteristics of the population under surveillance and the available capacities. Two probabilistic output-based measures are applied, i.e. the probability ('confidence') of detecting a case (surveillance sensitivity) and the probability that a population is free from disease (negative predictive value) [8]. While surveillance sensitivity allows achieving the targeted probability at the set design prevalence for different testing regimes, sample sizes and risk strata, the negative predictive value provides opportunities to combine information from multiple surveillance components and to take the value of historical information into account $[8,10,11]$. Consequently, heterogeneity in populations can be more adequately accounted for including various risk levels [9], and surveillance effectiveness can be quantified in populations that are too small to achieve the desired probability of detection when applying input-based standards [10-12]. Hence, these probabilistic approaches offer opportunities to lower costs while achieving the same target or achieve greater effectiveness at the same cost. Various analyses have been published demonstrating the superiority of output-based over input-based standards or risk-based over random sampling strategies. For instance, it was estimated that risk-based strategies reduced the cost to demonstrate freedom from infectious bovine rhinotraecheitis and enzootic bovine leucosis in the EU between 2002 and 2009 by six million Euros [13]. Output-based standards have been evaluated for 
example for Trichinella spp. [14], bovine herpes virus 1 [15], avian influenza virus [16], transmissible spongiform encephalopathies [17, 18], Mycobacterium bovis subsp. tuberculosis [19-22], Mycobacterium avium subsp. paratuberculosis [11, 23, 24], classical swine fever virus [25], bluetongue disease virus [26], and resistant pathogens [27]. However, output-based approaches need to be based on sound epidemiological knowledge including an evaluation of the epidemiological situation in the region, require close collaboration and exchange between scientists and policy makers [13] and need to be documented in a transparent manner to allow cross-country comparisons [28].

For early detection (or early warning surveillance), alternative approaches include for example sentinel, participatory and syndromic surveillance strategies [29]. Syndromic surveillance can be defined as 'surveillance of health indicators and diseases in defined populations in order to increase the likelihood of timely detection of undefined (new) or unexpected (exotic or re-emerging) threats' [2]. By detecting a disease early, potential devastating consequences of spread and thus economic impacts can be considerably minimised [28, 30]. Testing the same samples simultaneously for multiple hazards (multi-hazard surveillance) presents a general option to reduce surveillance costs and thus provides an important alternative to consider compared to targeting a single hazard only.

Although these approaches are increasingly promoted in scientific research, there is a lack of overview of AHS activities in EU countries and to what extent alternative strategies are already in place. Therefore, as part of the EU-FP7 RISKSUR project (20122015) (http://www.fp7-risksur.eu/), a survey was carried out to describe existing surveillance activities in EU countries, with the aim to identify gaps and opportunities. Furthermore, a better understanding of existing surveillance activities and differences between countries also contributed to inform the development of decision support tools for the design and evaluation of surveillance systems (http://webt ools.fp7-risksur.eu/).

\section{METHODS}

\section{Survey design}

The reference year was 2011 as this was the latest reporting period for which expenditures were accessible at the time of data collection (spring to autumn 2013). All seven 'partner countries' (France, FR;
Germany, DE; Spain, ES; Sweden, SE; Switzerland, $\mathrm{CH}$; The Netherlands, NL; United Kingdom, UK) of the RISKSUR Consortium as well as five selected 'nonpartner countries' (Bulgaria, BG; Czech Republic, CZ; Denmark, DK; Ireland, IE; Italy, IT) were included in the data collection process (subsequently referred to as study countries). Non-partner countries had been selected based on dissimilarity to partner countries regarding geographical region (i.e. targeting countries from Eastern and Southern Europe) and animal populations, production systems and hazards present, as well as the availability of a responsive official contact person. Furthermore, an in-country contact person had to be available to support data collection, especially due to language barriers and insufficient publicly accessible information on surveillance components, which would not have allowed for data collection otherwise.

Sequential numbers were randomly assigned to countries to preserve confidentiality. All hazards (e.g. pathogens, syndromes, antimicrobial resistance, animal welfare) and species were covered, regardless of whether surveillance was managed by the public or private sector. Surveillance associated with import or export testing was not considered as requirements depend on the trading partners of the respective country. Even though data were collected on active and passive components, only active surveillance components were considered in this analysis as data on passive surveillance were considered to be too similar between countries.

\section{Questionnaire design}

A questionnaire, comprising two sections with a total of 26 variables, was designed to collect information on surveillance components (see Supplementary Section S1 for a copy of the questionnaire and Supplementary Table S1 for examples). A surveillance component was defined as a single surveillance activity used to investigate the occurrence of one or more hazards or health events in a specified population, which has a selfcontained (i.e. conclusive and comprehensive in itself) surveillance protocol that focusses on a particular data source. The following key variables were used for analysis: targeted threat, disease or health event, target population, species and sector(s), geographical focus (local, national or regional), primary purpose, legal obligation, management (private, public or both), description of the component, study design (e.g. survey, continuous data collection), case definition (e.g. 
laboratory test for pathogen/toxins or host response, clinical signs, pathology, indirect indicators), risk-based sampling, and multi-hazard surveillance. Cost information is not presented in this paper as data gaps were too large to make meaningful inferences. Risk-based sampling was defined as preferentially sampling strata within the target population that are more likely to be exposed, affected, detected, become affected, transmit infection, or cause other consequences' [4]. For riskbased components, a post-hoc distinction was further made between targeted risk-based (focusing only on one sub-stratum of the population) and stratified riskbased (sampling intensity differs between population strata). Multi-hazard surveillance was defined as 'surveillance activities where samples collected for one disease agent are analysed for more than one purpose or for other disease agents, either in parallel or at a later stage'. For the surveillance definitions and characteristics used in the questionnaire, data collectors were referred to the final report from the International Conference on Animal Health Surveillance (ICAHS) in May 2011 [4].

\section{Data collection}

The questionnaire was circulated within the RISKSUR Consortium to collate feedback. Twentynine staff from RISKSUR partner countries collected the data in their own country and in collaboration with the assigned contact person in non-partner countries. All but one data collector were either trained veterinarians $(n=24)$ or animal scientists $(n=5)$. One external data collector who was temporarily employed in one non-partner country to ensure that language did not present a barrier was not working in the veterinary field. A training session was held with data collectors via Skype to review the protocol and thus standardize data collection. After approval by the RVC ethics committee (No. 2013 0071H: Ethical clearance for RISKSUR mapping), a database was developed in Microsoft Access (Microsoft Corp., USA) and distributed among data collectors. Information on surveillance components was predominantly collected through a grey literature search, including government or non-government reports, national legislations and other information, while scientific literature only sometimes provided indications of surveillance efforts. Representatives from public and private institutions were contacted to verify or complement information [31]. Finally, data were re-entered into a shared web-based SQL database.

\section{Data management}

Seven researchers from six institutes (APHA, CIRAD, FLI, RVC, SVA, UCM) spent 2 months on consistency checks of a subset of the database [34], which included discussions on standardized use of terminology and application to recorded components. A terminology working group was initiated to verify conclusions regarding means of data acquisition and surveillance purpose and objective [32]. Based on the results of this working group, the term 'surveillance objective' will be used instead of 'surveillance purpose' throughout the text. Prior to preparing this manuscript, a final consistency check was performed by a single investigator. This involved checking categorizations once again of those key variables, for which strong inconsistencies were identified during the initial consistency checks (i.e. surveillance objective, means of data acquisition, and risk-based sampling) and verification that components were consistently split according to the following criteria: (1) individual hazard(s) unless for unspecific components (e.g. meat inspection); (2) specified population: species and if applicable target sector; (3) data source: sampling point, case definition; (4) risk-based sampling; and (5) data collection method (means of data acquisition, study design).

\section{Data analysis}

Data were analysed in Stata statistical software release 14 (StataCorp LP, USA). A stacked bar chart of the number of components stratified by the sector managing the component (public, private, both, unknown) was created. Countries with obvious deficiencies in data completeness were excluded from subsequent analyses. Surveillance component was chosen as the level of analysis. The percentage and $95 \%$ confidence intervals of components (number of recorded components/total number of recorded components) were calculated for each variable using the cii command. Associations between each variable with the independent variables management, species and objective were explored using $r \times c$ contingency tables. Cells with low frequencies were collapsed or discarded if considered appropriate. The conditional probabilities (column percentages; tabulate command) and adjusted residuals (tabchi command) were reported. Any cell with adjusted residuals greater than \pm 1.96 was highlighted in bold, as they are more extreme than would be expected if the null hypothesis of independence was true [33].

Completeness of surveillance components per country was estimated by matching the components 
recorded by each country with all obligatory EU components identified in the dataset (see Supplementary Table S2) and dividing the achieved number by the total. An EU component was considered obligatory if it was compulsory for any EU Member State, not just relating to restriction zones or countries with eradication programmes in place.

\section{RESULTS}

\section{Descriptive results}

The dataset prior to the final consistency check included 738 active AHS components. Seventy-three components were excluded as they were combined with another component $(n=42)$, failed to meet the surveillance definition $(n=10)$, included insufficient information $(n$ $=8)$, related to export/import $(n=8)$, or were duplicates or considered erroneous $(n=5)$. Twenty-two components were added as recorded variables justified splitting the original component to achieve consistency in component splitting with other countries. Hence, the final dataset included 687 components.

The number of active AHS components recorded per country (median 57.5, range 10-105) stratified by the sector responsible for management (public, private or both) is shown in Supplementary Figure S1. In most countries, components were predominantly managed publicly [median across countries $65 \cdot 6 \%$, interquartile range (IQR) 47.5-75.7] with a smaller percentage being managed privately (median 19.8\%, IQR $9 \cdot 3-25 \cdot 6$ ) or in a public-private partnership (median 7.9\%, IQR 3.9-10.8).

Countries $5(n=13)$ and $11(n=10)$ were excluded from subsequent analyses as their data were considered to be too incomplete, thus resulting in 664 components in the final dataset. The remaining countries reported $34 \cdot 1-90 \cdot 2 \%$ of the 43 obligatory EU components identified in the dataset (median 59.8\%, IQR 48.2-84·1) (see Supplementary Table S2).

Components targeted 55 specific hazards (592 components), 21 hazard groups (e.g. wildlife diseases, emerging diseases) $(n=62)$ and three indicators (genetic, health, welfare) $(n=10)$. Supplementary Table S3 shows the number of components per hazard, the number of countries reporting at least one component for this hazard, and the median, minimum and maximum number of components for those latter countries. The most frequent hazards targeted by the hazard-specific components were Salmonella spp. (16.1\%), Brucella spp. (7·7\%), Mycobacterium tuberculosis (4.4\%), classical swine fever virus $(3 \cdot 9 \%)$, bluetongue disease virus (3.8\%), avian influenza virus, scrapie and Trichinella spp. (3.6\% each), and Aujeszky's disease virus and enzootic bovine leucosis virus $(3 \cdot 3 \%$ each).

Descriptive results for all study countries and stratified by partner and non-partner countries are presented in Table 1. Categories of three variables significantly differed between partner and non-partner countries, i.e. legal requirement, management and risk-based sampling.

Cattle were the most frequent species targeted by components $(26 \cdot 7 \%)$, followed by pigs $(17 \cdot 5 \%)$, poultry $(16.0 \%)$, and small ruminants $(11.9 \%)$. Country $9 \mathrm{did}$ not record any wildlife components and countries 1 and 7 only one general wildlife component. The category 'other species' covered fish (13 components), insect vectors $(n=9)$, and bees $(n=3)$. Fewer than five components were recorded for molluscs, shellfish or crustaceans $(n=4)$, animal feed $(n=4)$, and pets $(n=$ $1)$. Most components were implemented at the national level $(89 \cdot 4 \%)$ and were based on EU regulations $(68.4 \%)$. Twelve percent of components were based on additional national requirements.

The most commonly assigned surveillance objective was demonstrating freedom from disease $(43 \cdot 8 \%)$, followed by case detection $(26 \cdot 8 \%)$, prevalence estimation $(19 \cdot 7 \%)$, and early detection $(9 \cdot 8 \%)$. The sampling point was recorded as farm, abattoir, and insemination centre for $48 \cdot 6 \%, 21 \cdot 4 \%$, and $15 \cdot 6 \%$ of components, respectively. Data were usually recorded to be collected continuously $(56 \cdot 8 \%)$ or via repeated (usually annual) surveys $(39 \cdot 2 \%)$. Case reports $(n=15)$, sentinel surveillance $(n=8)$, participatory surveillance $(n=2)$, and event-based surveillance $(n=1)$ were recorded under 'other study designs'. Laboratory diagnosis (direct, indirect or both) was the most common case definition. Active clinical surveillance $(n=12)$, i.e. routine inspection by the competent authority without prior notification of abnormal signs by farmers, targeted bluetongue disease, classical swine fever and emerging diseases in more than one country. Most 'other case definitions' contained multiple case definitions including others than laboratory detection (e.g. indirect indicators, risk factors). Risk-based sampling and multi-hazard surveillance were recorded for $57 \cdot 1 \%$ and $37 \cdot 3 \%$ of components, respectively.

\section{Bivariate results: management}

Components managed in a public-private partnership were more likely recorded (than what would be 
Table 1. Percentage of active surveillance components (number of recorded components/total number of recorded components) and 95\% confidence intervals (CI) by surveillance design variables for all ten study countries, stratified by whether countries were partners of the EU-FP7 RISKSUR project or not. Components comprised active surveillance components implemented in 2011. Categories, for which confidence intervals between partner and non-partner countries did not overlap, are highlighted in grey

\begin{tabular}{|c|c|c|c|c|}
\hline Variable $(n)^{*}$ & Category & All $(n=664)$ & Partner $(n=421)$ & Non-partner $(n=243)$ \\
\hline \multirow[t]{8}{*}{ Species $(664 / 421 / 243)$} & Cattle & $26 \cdot 7(23 \cdot 3-30 \cdot 2)$ & $26 \cdot 8(22 \cdot 7-31 \cdot 3)$ & $26 \cdot 3(20 \cdot 9-32 \cdot 3)$ \\
\hline & Pigs & $17 \cdot 5(14 \cdot 7-20 \cdot 6)$ & $17 \cdot 8(14 \cdot 3-21 \cdot 8)$ & $16 \cdot 9(12 \cdot 4-22 \cdot 2)$ \\
\hline & Poultry & $16 \cdot 0(13 \cdot 3-19 \cdot 0)$ & $14 \cdot 0(10 \cdot 8-17 \cdot 7)$ & $19 \cdot 3(14 \cdot 6-24 \cdot 9)$ \\
\hline & Small ruminants & $11 \cdot 9(9 \cdot 5-14 \cdot 6)$ & $11 \cdot 9(8 \cdot 9-15 \cdot 4)$ & $11 \cdot 9(8 \cdot 1-16 \cdot 7)$ \\
\hline & Wildlife & $10 \cdot 2(8 \cdot 0-12 \cdot 8)$ & $11 \cdot 4(8 \cdot 5-14 \cdot 8)$ & $8 \cdot 2(5 \cdot 1-12 \cdot 4)$ \\
\hline & Equidae & $6 \cdot 9(5 \cdot 1-9 \cdot 1)$ & $7 \cdot 4(5 \cdot 1-10 \cdot 3)$ & $6 \cdot 2(3 \cdot 5-10 \cdot 0)$ \\
\hline & Multi & $5 \cdot 4(3 \cdot 8-7 \cdot 4)$ & $5 \cdot 0(3 \cdot 1-7 \cdot 5)$ & $6 \cdot 2(3 \cdot 5-10 \cdot 0)$ \\
\hline & Other & $5 \cdot 4(3 \cdot 8-7 \cdot 4)$ & $5 \cdot 7(3 \cdot 7-8 \cdot 4)$ & $4 \cdot 9(2 \cdot 6-8 \cdot 5)$ \\
\hline \multirow[t]{3}{*}{ Management (615/418/197) } & Public & $65 \cdot 4(61 \cdot 5-69 \cdot 1)$ & $64 \cdot 4(59 \cdot 6-68 \cdot 9)$ & $67 \cdot 5(60 \cdot 5-74 \cdot 0)$ \\
\hline & Private & $24 \cdot 7(21 \cdot 4-28 \cdot 3)$ & $23 \cdot 2(19 \cdot 2-27 \cdot 6)$ & $27 \cdot 9(21 \cdot 8-34 \cdot 7)$ \\
\hline & Both & $9 \cdot 9(7 \cdot 7-12 \cdot 6)$ & $12 \cdot 4(9 \cdot 4-16 \cdot 0)$ & $4 \cdot 6(2 \cdot 1-8 \cdot 5)$ \\
\hline \multirow[t]{3}{*}{ Area $(662 / 421 / 241)$} & National & $89 \cdot 4(86 \cdot 8-91 \cdot 7)$ & $87 \cdot 4(83 \cdot 9-90 \cdot 4)$ & $92 \cdot 9(88 \cdot 9-95 \cdot 8)$ \\
\hline & Regional & $8 \cdot 6(6 \cdot 6-11 \cdot 0)$ & $9 \cdot 7(7 \cdot 1-13 \cdot 0)$ & $6 \cdot 6(3 \cdot 8-10 \cdot 6)$ \\
\hline & Local & $2 \cdot 0(1 \cdot 0-3 \cdot 3)$ & $2 \cdot 9(1 \cdot 5-4 \cdot 9)$ & $0 \cdot 4(0-2 \cdot 3)$ \\
\hline \multirow[t]{5}{*}{ Obligation (645/417/228) } & EU & $68 \cdot 4(64 \cdot 6-71 \cdot 9)$ & $58 \cdot 5(53 \cdot 6-63 \cdot 3)$ & $86 \cdot 4(81 \cdot 3-90 \cdot 6)$ \\
\hline & National & $11 \cdot 6(9 \cdot 3-14 \cdot 4)$ & $12 \cdot 7(9 \cdot 7-16 \cdot 3)$ & $9 \cdot 6(6 \cdot 1-14 \cdot 2)$ \\
\hline & Regional & $2 \cdot 2(1 \cdot 2-3 \cdot 6)$ & $2 \cdot 6(1 \cdot 3-4 \cdot 7)$ & $1 \cdot 3(0 \cdot 3-3 \cdot 8)$ \\
\hline & Private & $7 \cdot 9(5.9-10.3)$ & $11 \cdot 8(8 \cdot 8-15 \cdot 2)$ & $0 \cdot 9(0 \cdot 1-3 \cdot 1)$ \\
\hline & None & $9 \cdot 9(7.7-12.5)$ & $14 \cdot 4(11 \cdot 2-18 \cdot 1)$ & $1 \cdot 8(0 \cdot 5-4 \cdot 4)$ \\
\hline \multirow[t]{4}{*}{ Objective (656/417/239) } & Disease freedom & $43 \cdot 8(39.9-47.6)$ & $41 \cdot 7(36 \cdot 9-46 \cdot 6)$ & $47 \cdot 3(40 \cdot 8-53 \cdot 8)$ \\
\hline & Case detection & $26 \cdot 8(23 \cdot 5-30 \cdot 4)$ & $26 \cdot 9(22 \cdot 7-31 \cdot 4)$ & $26 \cdot 8(21 \cdot 3-32 \cdot 9)$ \\
\hline & Prevalence estimation & $19 \cdot 7(16 \cdot 7-22 \cdot 9)$ & $22 \cdot 5(18 \cdot 6-26 \cdot 9)$ & $14 \cdot 6(10 \cdot 4-19 \cdot 8)$ \\
\hline & Early detection & $9 \cdot 8(7 \cdot 6-12 \cdot 3)$ & $8 \cdot 9(6 \cdot 3-12 \cdot 0)$ & $11 \cdot 3(7 \cdot 6-16 \cdot 0)$ \\
\hline \multirow[t]{6}{*}{ Sampling point $(646 / 405 / 241)$} & Farm & $48 \cdot 6(44 \cdot 7-52 \cdot 5)$ & $47 \cdot 2(42 \cdot 2-52 \cdot 2)$ & $51 \cdot 0(44 \cdot 5-57 \cdot 5)$ \\
\hline & Abattoir & $21 \cdot 4(18 \cdot 3-24 \cdot 7)$ & $23 \cdot 0(19 \cdot 0-27 \cdot 4)$ & $18 \cdot 7(14 \cdot 0-24 \cdot 2)$ \\
\hline & Insemination & $15 \cdot 6(12 \cdot 9-18 \cdot 7)$ & $14 \cdot 8(11 \cdot 5-18 \cdot 7)$ & $17 \cdot 0(12 \cdot 5-22 \cdot 4)$ \\
\hline & Wild & $8 \cdot 5(6 \cdot 5-10 \cdot 9)$ & $8 \cdot 4(5 \cdot 9-11 \cdot 5)$ & $8 \cdot 7(5 \cdot 5-13 \cdot 0)$ \\
\hline & Rendering & $3 \cdot 7(2 \cdot 4-5 \cdot 5)$ & $4 \cdot 0(2 \cdot 3-6 \cdot 3)$ & $3 \cdot 3(1 \cdot 4-6 \cdot 4)$ \\
\hline & Other & $2 \cdot 2(1 \cdot 2-3 \cdot 6)$ & $2 \cdot 7(1 \cdot 4-4 \cdot 8)$ & $1 \cdot 2(0 \cdot 3-3 \cdot 6)$ \\
\hline \multirow[t]{3}{*}{ Study type $(655 / 415 / 240)$} & Continous & $56 \cdot 8(52 \cdot 9-60 \cdot 6)$ & $59 \cdot 0(54 \cdot 1-63 \cdot 8)$ & $52 \cdot 9(46 \cdot 4-59 \cdot 4)$ \\
\hline & Survey & $39 \cdot 2(35 \cdot 5-43 \cdot 1)$ & $35 \cdot 9(31 \cdot 3-40 \cdot 7)$ & $45 \cdot 0(38 \cdot 6-51 \cdot 5)$ \\
\hline & Other & $4 \cdot 0(2 \cdot 6-5 \cdot 8)$ & $5 \cdot 1(3 \cdot 2-7 \cdot 6)$ & $2 \cdot 1(0 \cdot 7-4 \cdot 8)$ \\
\hline \multirow[t]{5}{*}{ Case definition $(655 / 417 / 238)$} & Lab: Pathogen/toxin & $42 \cdot 9(39 \cdot 1-46 \cdot 8)$ & $45 \cdot 3(40 \cdot 5-50 \cdot 2)$ & $38 \cdot 7(32 \cdot 4-45 \cdot 2)$ \\
\hline & Lab: Host response & $37 \cdot 9(34 \cdot 1-41 \cdot 7)$ & $34 \cdot 8(30 \cdot 2-39 \cdot 6)$ & $43 \cdot 3(36 \cdot 9-49 \cdot 8)$ \\
\hline & Both & $7 \cdot 6(5 \cdot 7-9 \cdot 9)$ & $5 \cdot 5(3 \cdot 5-8 \cdot 2)$ & $11 \cdot 3(7 \cdot 6-16 \cdot 1)$ \\
\hline & Clinical/pathological & $2 \cdot 9(1 \cdot 8-4 \cdot 5)$ & $3 \cdot 8(2 \cdot 2-6 \cdot 2)$ & $1 \cdot 3(0 \cdot 3-3 \cdot 6)$ \\
\hline & Other & $8 \cdot 7(6 \cdot 7-11 \cdot 1)$ & $10 \cdot 6(7 \cdot 8-13 \cdot 9)$ & $5 \cdot 5(2 \cdot 9-9 \cdot 2)$ \\
\hline \multirow[t]{2}{*}{ Risk-based (641/413/228) } & Yes & $57 \cdot 1(53 \cdot 2-61 \cdot 0)$ & $52 \cdot 1(47 \cdot 1-57 \cdot 0)$ & $66 \cdot 2(59 \cdot 7-72 \cdot 3)$ \\
\hline & No & $42 \cdot 9(39 \cdot 0-46 \cdot 8)$ & $47 \cdot 9(43 \cdot 0-52 \cdot 9)$ & $33 \cdot 8(27 \cdot 7-40 \cdot 3)$ \\
\hline \multirow[t]{2}{*}{ Multi-hazard (579/396/183) } & Yes & $37 \cdot 3(33 \cdot 4-41 \cdot 4)$ & $40 \cdot 4(35 \cdot 5-45 \cdot 4)$ & $30 \cdot 6(24 \cdot 0-37 \cdot 8)$ \\
\hline & No & $62 \cdot 7(58 \cdot 6-66 \cdot 6)$ & $59 \cdot 6(54 \cdot 6-64 \cdot 5)$ & $69 \cdot 4(62 \cdot 2-76 \cdot 0)$ \\
\hline
\end{tabular}

* Number of observations for those categories listed for the respective variable. The difference in observations compared to the number provided below the heading of 3rd to 5 th columns comprises the number of missing observations per category.

expected if the variables were independent) by partner $(85 \cdot 3 \%)$ than non-partner $(14 \cdot 8 \%)$ countries (Table 2$)$. Privately managed components more frequently targeted cattle $(46 \cdot 6 \%)$ and less frequently small ruminants $(8 \cdot 4 \%)$ than public components $(34 \cdot 0 \%$ and $21 \cdot 5 \%$, respectively). Sixty percent of private components compared to $39 \cdot 9 \%$ of public components aimed to demonstrate freedom from disease. In contrast, prevalence estimation and early detection were more frequently recorded as surveillance objective for public $(22 \cdot 7 \%$ 
Table 2. Column percentages and adjusted residuals (in parentheses)* of management vs. variables for active surveillance components in 2011 recorded by 10 study countries $(n=615)$

\begin{tabular}{|c|c|c|c|c|}
\hline Variable $\dagger$ & Category & Public & Private & Both \\
\hline \multirow[t]{2}{*}{ Partner country $(n=615)$} & Yes & $66 \cdot 9(-0 \cdot 77)$ & $63 \cdot 8(-1 \cdot 26)$ & $85 \cdot 3(3 \cdot 05)$ \\
\hline & No & $33 \cdot 1(0 \cdot 77)$ & $36 \cdot 2(1 \cdot 26)$ & $14.8(-3.05)$ \\
\hline \multirow[t]{4}{*}{ Species group $(n=441)$} & Cattle & $34 \cdot 0(-2 \cdot 43)$ & $46 \cdot 6(2 \cdot 25)$ & $42 \cdot 2(0 \cdot 53)$ \\
\hline & Pigs & $23 \cdot 8(0.39)$ & $22.9(-0.07)$ & $20 \cdot 0(-0.53)$ \\
\hline & Poultry & $20 \cdot 8(-0 \cdot 35)$ & $22 \cdot 1(0 \cdot 27)$ & $22 \cdot 2(0 \cdot 16)$ \\
\hline & Small ruminants & $21.5(3.09)$ & $8 \cdot 4(-3 \cdot 13)$ & $15 \cdot 6(-0 \cdot 27)$ \\
\hline \multirow[t]{2}{*}{ Geographical focus ( $n=595)$} & National & $89 \cdot 3(-3 \cdot 27)$ & $97 \cdot 2(2 \cdot 70)$ & $96 \cdot 5(1 \cdot 33)$ \\
\hline & Regional & $10 \cdot 7(3 \cdot 27)$ & $2 \cdot 8(-2 \cdot 70)$ & $3 \cdot 5(-1 \cdot 33)$ \\
\hline \multirow[t]{3}{*}{ Obligation $(n=610)$} & $\mathrm{EU}$ & $72 \cdot 3(2 \cdot 85)$ & $65 \cdot 8(-0.79)$ & $49 \cdot 2(-3 \cdot 40)$ \\
\hline & National or regional & $16 \cdot 4(2 \cdot 37)$ & $5.9(-3 \cdot 29)$ & $18.0(0.97)$ \\
\hline & Private or voluntary & $11 \cdot 3(-5 \cdot 63)$ & $28 \cdot 3(3.95)$ & $32 \cdot 8(3 \cdot 25)$ \\
\hline \multirow[t]{4}{*}{ Surveillance objective $(n=608)$} & Disease freedom & $39 \cdot 9(-3.00)$ & $59 \cdot 9(4 \cdot 48)$ & $32 \cdot 7(-1 \cdot 80)$ \\
\hline & Case detection & $25.9(-1.05)$ & $28 \cdot 3(0 \cdot 32)$ & $34 \cdot 6(1 \cdot 26)$ \\
\hline & Prevalence estimation & $22 \cdot 7(3 \cdot 00)$ & $8 \cdot 6(-3 \cdot 86)$ & $23 \cdot 6(0 \cdot 87)$ \\
\hline & Early detection & $11 \cdot 5(2 \cdot 68)$ & $3 \cdot 3(-2 \cdot 92)$ & $9 \cdot 1(-0 \cdot 03)$ \\
\hline \multirow[t]{4}{*}{ Sampling point $(n=535)$} & Farm & $58 \cdot 0(1 \cdot 48)$ & $43 \cdot 8(-3 \cdot 32)$ & $70 \cdot 0(2 \cdot 40)$ \\
\hline & Abattoir & $30 \cdot 5(5 \cdot 27)$ & $8 \cdot 3(-4 \cdot 89)$ & $16 \cdot 7(-1 \cdot 24)$ \\
\hline & Rendering plant & $5 \cdot 1(1 \cdot 52)$ & $0 \cdot 0(-2 \cdot 91)$ & $8 \cdot 3(1.75)$ \\
\hline & Insemination centre & $6 \cdot 3(-8 \cdot 58)$ & $47 \cdot 9(11 \cdot 31)$ & $5 \cdot 0(-2 \cdot 69)$ \\
\hline \multirow[t]{3}{*}{ Study design $(n=609)$} & Continuous & $61 \cdot 2(4 \cdot 42)$ & $39 \cdot 5(-4 \cdot 35)$ & $50 \cdot 0(-0 \cdot 75)$ \\
\hline & Survey & $33 \cdot 6(-5 \cdot 16)$ & $58 \cdot 6(5 \cdot 06)$ & $46 \cdot 6(0 \cdot 90)$ \\
\hline & Other & $5 \cdot 3(1.67)$ & $2 \cdot 0(-1 \cdot 62)$ & $3 \cdot 5(-0 \cdot 33)$ \\
\hline \multirow[t]{4}{*}{ Case definition $(n=556)$} & Direct & $66 \cdot 1(2 \cdot 77)$ & $37 \cdot 4(-3 \cdot 04)$ & $50 \cdot 1(1 \cdot 01)$ \\
\hline & Serological & $23 \cdot 2(-2 \cdot 64)$ & $54 \cdot 7(4 \cdot 21)$ & $36 \cdot 3(-2 \cdot 15)$ \\
\hline & Both & $7 \cdot 1(-0 \cdot 42)$ & $3 \cdot 6(-2 \cdot 44)$ & $10 \cdot 8(2 \cdot 48)$ \\
\hline & Clinical/pathological & $3 \cdot 6(0 \cdot 15)$ & $4 \cdot 3(0 \cdot 83)$ & $2 \cdot 8(-0 \cdot 85)$ \\
\hline \multirow[t]{2}{*}{ Risk-based $(n=600)$} & Yes & $53 \cdot 1(-2 \cdot 42)$ & $66 \cdot 0(2 \cdot 66)$ & $56 \cdot 7(0.00)$ \\
\hline & No & $46 \cdot 9(2 \cdot 42)$ & $34 \cdot 0(-2 \cdot 66)$ & $43 \cdot 3(0 \cdot 00)$ \\
\hline \multirow[t]{2}{*}{ Multi-hazard $(n=548)$} & Yes & $28 \cdot 6(-6 \cdot 58)$ & $68 \cdot 1(8 \cdot 15)$ & $31 \cdot 7(-1 \cdot 21)$ \\
\hline & No & $71 \cdot 4(6 \cdot 58)$ & $31 \cdot 9(-8 \cdot 15)$ & $68 \cdot 3(1 \cdot 21)$ \\
\hline
\end{tabular}

* Adjusted residuals greater than $\pm 1 \cdot 96$ are highlighted in bold as they are more extreme than what would be expected if the null hypothesis of independence was true.

$\dagger$ The difference in the number of components compared to the total number indicated in the heading comprises missing or unknown observations.

and $11 \cdot 5 \%)$ than for private $(8 \cdot 6 \%$ and $3 \cdot 3 \%)$ components. Continuous data collection was more commonly managed publicly $(61 \cdot 2 \%)$ than privately $(39 \cdot 5 \%)$, while repeated surveys were more predominant for private $(58.6 \%)$ than public $(33.6 \%)$ components. Risk-based strategies and multi-hazard surveillance were more often recorded for privately $(66.0 \%$ and $68.1 \%$, respectively) than for publicly ( $53 \cdot 1 \%$ and $28 \cdot 6 \%$, respectively) managed components.

\section{Bivariate results: species}

Components targeting pigs were more likely based on additional national regulations $(23.0 \%)$ than those targeting poultry $(6 \cdot 1 \%)$ (Table 3$)$. The objective of demonstrating freedom from disease was more likely recorded for cattle and pigs $(59 \cdot 1 \%$ and $58.6 \%$, respectively) than for poultry $(15 \cdot 1 \%)$. Case detection was more frequently recorded for poultry $(65 \cdot 1 \%)$ compared to the other three species groups, while prevalence estimation was more likely recorded for small ruminants $(35 \cdot 4 \%)$ compared to poultry $(8 \cdot 5 \%)$. Farm was more frequently recorded as sampling point for poultry $(89 \cdot 1 \%)$ than for cattle $(46 \cdot 0 \%)$ and pigs $(37 \cdot 2 \%)$, while abattoir was more common for surveillance components targeting pigs $(38 \cdot 1 \%)$ compared to poultry $(10 \cdot 9 \%)$. Risk-based strategies were relatively seldomly recorded for pigs $(50 \cdot 4 \%)$, while multi-hazard surveillance was relatively frequently recorded for pigs $(59 \cdot 0 \%)$ and cattle $(44 \cdot 0 \%)$. In contrast, multi-hazard surveillance was relatively uncommon for poultry $(8 \cdot 1 \%)$. 
Table 3. Column percentages and adjusted residuals (in parentheses)* of livestock species vs. variables for active surveillance components in 2011 recorded by 10 study countries $(n=487)$

\begin{tabular}{|c|c|c|c|c|c|}
\hline Variable $\dagger$ & Category & Cattle & Pigs & Poultry & Small ruminants \\
\hline \multirow[t]{2}{*}{ Partner country $(n=487)$} & Yes & $64 \cdot 5(0 \cdot 75)$ & $64 \cdot 7(0 \cdot 57)$ & $55 \cdot 7(-1 \cdot 63)$ & $63 \cdot 3(0 \cdot 17)$ \\
\hline & No & $35 \cdot 5(-0.75)$ & $35 \cdot 3(-0 \cdot 57)$ & $44.3(1.63)$ & $36 \cdot 7(-0 \cdot 17)$ \\
\hline \multirow[t]{2}{*}{ Geographical focus ( $n=474)$} & National & $92 \cdot 9(-0 \cdot 12)$ & $89.0(-1.89)$ & $100 \cdot 0(3 \cdot 20)$ & $89.6(-1.29)$ \\
\hline & Regional & $7 \cdot 1(0 \cdot 12)$ & $11.0(1.89)$ & $0 \cdot 0(-3 \cdot 20)$ & $10 \cdot 4(1.29)$ \\
\hline \multirow[t]{3}{*}{ Obligation $(n=472)$} & $\mathrm{EU}$ & $68 \cdot 1(-0 \cdot 51)$ & $59 \cdot 3(-2 \cdot 70)$ & $86 \cdot 7(4 \cdot 17)$ & $65 \cdot 8(-0 \cdot 78)$ \\
\hline & National or regional & $11.5(-0.92)$ & $23 \cdot 0(3 \cdot 46)$ & $6 \cdot 1(-2 \cdot 36)$ & $12 \cdot 7(-0 \cdot 20)$ \\
\hline & Private or voluntary & $20 \cdot 3(1.45)$ & $17 \cdot 7(0 \cdot 17)$ & $7 \cdot 1(-2 \cdot 96)$ & $21 \cdot 5(1.13)$ \\
\hline \multirow[t]{4}{*}{ Surveillance objective $(n=487)$} & Disease freedom & $59 \cdot 1(4 \cdot 36)$ & $58 \cdot 6(2.97)$ & $15 \cdot 1(-7 \cdot 35)$ & $41.8(-0.94)$ \\
\hline & Case detection & $14 \cdot 5(-4 \cdot 71)$ & $17 \cdot 2(-2 \cdot 59)$ & $65 \cdot 1(10 \cdot 18)$ & $16 \cdot 5(-2 \cdot 21)$ \\
\hline & Prevalence estimation & $21.0(0.83)$ & $14.7(-1.39)$ & $8 \cdot 5(-3 \cdot 14)$ & $35 \cdot 4(4 \cdot 04)$ \\
\hline & Early detection & $5.4(-1.57)$ & $9 \cdot 5(0.77)$ & $11 \cdot 3(1 \cdot 53)$ & $6 \cdot 3(-0 \cdot 53)$ \\
\hline \multirow[t]{4}{*}{ Sampling point $(n=456)$} & Farm & $46 \cdot 0(-3 \cdot 07)$ & $37 \cdot 2(-4 \cdot 40)$ & $89 \cdot 1(7 \cdot 36)$ & $61 \cdot 3(1 \cdot 20)$ \\
\hline & Abattoir & $20 \cdot 5(-0 \cdot 60)$ & $38 \cdot 1(4 \cdot 78)$ & $10.9(-2 \cdot 87)$ & $14 \cdot 7(-1 \cdot 66)$ \\
\hline & Rendering plant & $6.3(1.33)$ & $0.9(-2 \cdot 18)$ & $0 \cdot 0(-2 \cdot 36)$ & $12.0(3.34)$ \\
\hline & Insemination centre & $27 \cdot 3(3 \cdot 87)$ & $23 \cdot 9(1 \cdot 73)$ & $0 \cdot 0(-5 \cdot 10)$ & $12 \cdot 0(-1.57)$ \\
\hline \multirow[t]{3}{*}{ Study design $(n=481)$} & Continuous & $51 \cdot 6(-0 \cdot 55)$ & $55 \cdot 7(0 \cdot 60)$ & $60 \cdot 4(1 \cdot 67)$ & $43 \cdot 4(-1 \cdot 87)$ \\
\hline & Survey & $44 \cdot 6(0 \cdot 24)$ & $41 \cdot 7(-0.53)$ & $37 \cdot 7(-1.44)$ & $54.0(1.93)$ \\
\hline & Other & $3.8(0.92)$ & $2 \cdot 6(-0.22)$ & $1.9(-0.71)$ & $2 \cdot 6(-0 \cdot 16)$ \\
\hline \multirow[t]{4}{*}{ Case definition $(n=452)$} & Direct & $36 \cdot 0(-2 \cdot 20)$ & $24 \cdot 6(-4 \cdot 48)$ & $71 \cdot 8(6 \cdot 75)$ & $44 \cdot 9(0 \cdot 38)$ \\
\hline & Serological & $55 \cdot 3(2 \cdot 30)$ & $60 \cdot 9(3 \cdot 11)$ & $24 \cdot 3(-5 \cdot 49)$ & $46 \cdot 2(-0 \cdot 36)$ \\
\hline & Both & $7 \cdot 5(0 \cdot 67)$ & $10 \cdot 0(1 \cdot 76)$ & $1.9(-2 \cdot 11)$ & $5 \cdot 1(-0 \cdot 51)$ \\
\hline & Clinical/pathological & $1 \cdot 2(-1 \cdot 39)$ & $4 \cdot 6(1 \cdot 42)$ & $1.9(-0.51)$ & $3.9(0.72)$ \\
\hline \multirow[t]{2}{*}{ Risk-based $(n=477)$} & Yes & $65 \cdot 6(1 \cdot 23)$ & $50 \cdot 4(-2 \cdot 95)$ & $62 \cdot 3(0 \cdot 05)$ & $71 \cdot 1(1 \cdot 76)$ \\
\hline & No & $34 \cdot 4(-1 \cdot 23)$ & $49 \cdot 6(2 \cdot 95)$ & $37 \cdot 7(-0.05)$ & $29 \cdot 0(-1 \cdot 76)$ \\
\hline \multirow[t]{2}{*}{ Multi-hazard $(n=426)$} & Yes & $44 \cdot 0(2 \cdot 61)$ & $59 \cdot 0(5 \cdot 44)$ & $8 \cdot 1(-6 \cdot 64)$ & $25 \cdot 0(-2.09)$ \\
\hline & No & $56 \cdot 0(-2 \cdot 61)$ & $41 \cdot 0(-5 \cdot 44)$ & $91.9(6 \cdot 64)$ & $75 \cdot 0(2 \cdot 09)$ \\
\hline
\end{tabular}

* Adjusted residuals greater than $\pm 1 \cdot 96$ are highlighted in bold as they are more extreme than what would be expected if the null hypothesis of independence was true.

$\dagger$ The difference in the number of components compared to the total number indicated in the heading comprises missing or unknown observations.

\section{Bivariate results: surveillance objective}

The objective prevalence estimation was more frequently recorded by partner $(72.9 \%)$ than by nonpartner $(27 \cdot 1 \%)$ countries (Table 4). Components were more commonly implemented at the national level for the objective of demonstrating disease freedom than for early detection and at the regional level vice versa. Seventy-six percent of components with the objective of demonstrating freedom from disease were regulated by the EU compared to $45.5 \%$ of components aimed at early detection. Components aimed at early detection were more frequently regulated by national regulations or voluntary programmes than components with other objectives. At the farm level, the most frequent objectives were case detection $(79 \cdot 4 \%)$ and early detection $(71.7 \%)$. The objective of annual surveys at insemination centres was consistently categorized as demonstrating freedom from disease $(100 \%)$. Components aimed at demonstrating freedom from disease more commonly applied risk-based sampling (63.3\%) and multi-hazard surveillance $(59 \cdot 5 \%)$ compared to other objectives (risk-based: 44.2-57.9\%; multi-hazard: $12.8-35.1 \%)$.

\section{Risk-based sampling}

The most frequent risk factors were production type (breeder, grower), age, region, herd size, and time period (Table 5). Of these top five risk factors, production type, age, and herd size predominantly included targeted risk-based strategies, where only a single stratum is under surveillance (e.g. only breeders or only animals above a certain age). In contrast, for the risk factor region, the component description mostly indicated stratified risk-based sampling, e.g. targeting regions with different sampling intensity depending on differences in epidemiological situation or risk of 
Table 4. Column percentages and adjusted residuals (in parentheses)* of surveillance objective vs. variables for active surveillance components in 2011 recorded by 10 study countries $(n=656)$

\begin{tabular}{|c|c|c|c|c|c|}
\hline Variable $\dagger$ & Category & Case detection & Early detection & Disease freedom & $\begin{array}{l}\text { Prevalence } \\
\text { estimation }\end{array}$ \\
\hline \multirow[t]{2}{*}{ Partner country $(n=656)$} & Yes & $63 \cdot 6(0 \cdot 02)$ & $57 \cdot 8(-1 \cdot 01)$ & $60 \cdot 6(-1 \cdot 38)$ & $72 \cdot 9(2 \cdot 45)$ \\
\hline & No & $36 \cdot 4(-0 \cdot 02)$ & $42 \cdot 2(1 \cdot 01)$ & $39 \cdot 4(1 \cdot 38)$ & $27 \cdot 1(-2 \cdot 45)$ \\
\hline \multirow[t]{2}{*}{ Geographical focus $(n=636)$} & National & $91 \cdot 2(-0 \cdot 36)$ & $84 \cdot 4(-2 \cdot 29)$ & $95 \cdot 7(3 \cdot 11)$ & $88 \cdot 0(-1 \cdot 74)$ \\
\hline & Regional & $8 \cdot 8(0 \cdot 36)$ & $15 \cdot 6(2 \cdot 29)$ & $4 \cdot 3(-3 \cdot 11)$ & $12 \cdot 0(1 \cdot 74)$ \\
\hline \multirow[t]{3}{*}{ Obligation $(n=636)$} & $\mathrm{EU}$ & $65 \cdot 5(-0 \cdot 96)$ & $45 \cdot 5(-3 \cdot 83)$ & $76 \cdot 4(3 \cdot 86)$ & $64 \cdot 6(-1 \cdot 04)$ \\
\hline & National or regional & $13 \cdot 2(-0 \cdot 21)$ & $23 \cdot 6(2 \cdot 25)$ & $11 \cdot 4(-1 \cdot 47)$ & $15 \cdot 0(0 \cdot 47)$ \\
\hline & Private or voluntary & $21 \cdot 3(1 \cdot 35)$ & $30 \cdot 9(2 \cdot 63)$ & $12 \cdot 1(-3 \cdot 37)$ & $20 \cdot 5(0 \cdot 84)$ \\
\hline \multirow[t]{4}{*}{ Sampling point $(n=574)$} & Farm & $79 \cdot 4(7 \cdot 28)$ & $71 \cdot 7(2 \cdot 37)$ & $40 \cdot 4(-6 \cdot 31)$ & $46.9(-1.94)$ \\
\hline & Abattoir & $19 \cdot 4(-1 \cdot 40)$ & $23 \cdot 9(0 \cdot 10)$ & $19 \cdot 2(-2 \cdot 09)$ & $38 \cdot 1(4 \cdot 12)$ \\
\hline & Rendering plant & $1 \cdot 3(-2 \cdot 18)$ & $4 \cdot 4(0 \cdot 06)$ & $1 \cdot 2(-3 \cdot 22)$ & $15 \cdot 0(6 \cdot 44)$ \\
\hline & Insemination centre & $0 \cdot 0(-6 \cdot 84)$ & $0 \cdot 0(-3 \cdot 25)$ & $39 \cdot 2(12 \cdot 31)$ & $0 \cdot 0(-5 \cdot 45)$ \\
\hline \multirow[t]{3}{*}{ Study design $(n=649)$} & Continuous & $69 \cdot 9(4 \cdot 01)$ & $73 \cdot 4(2 \cdot 80)$ & $36 \cdot 3(-9 \cdot 42)$ & $77 \cdot 3(5 \cdot 19)$ \\
\hline & Survey & $28 \cdot 3(-3 \cdot 36)$ & $0 \cdot 0(-6 \cdot 74)$ & $62 \cdot 7(10 \cdot 92)$ & $20 \cdot 3(-4 \cdot 83)$ \\
\hline & Other & $1 \cdot 7(-1 \cdot 78)$ & $26 \cdot 6(9 \cdot 69)$ & $1 \cdot 1(-3 \cdot 38)$ & $2 \cdot 3(-1 \cdot 07)$ \\
\hline \multirow[t]{4}{*}{ Case definition $(n=592)$} & Direct & $68 \cdot 6(6 \cdot 36)$ & $24 \cdot 0(-3 \cdot 38)$ & $27 \cdot 6(-8 \cdot 64)$ & $72 \cdot 8(6 \cdot 20)$ \\
\hline & Serological & $24 \cdot 4(-5 \cdot 17)$ & $44 \cdot 0(0 \cdot 32)$ & $60 \cdot 7(8 \cdot 53)$ & $20 \cdot 2(-5 \cdot 23)$ \\
\hline & Both & $4 \cdot 5(-1.93)$ & $20 \cdot 0(3 \cdot 22)$ & $10 \cdot 3(1 \cdot 80)$ & $2 \cdot 6(-2 \cdot 38)$ \\
\hline & $\begin{array}{l}\text { Clinical/ } \\
\text { pathological }\end{array}$ & $2 \cdot 6(-0 \cdot 53)$ & $12 \cdot 0(3 \cdot 69)$ & $1 \cdot 5(-2 \cdot 21)$ & $4 \cdot 4(0 \cdot 79)$ \\
\hline \multirow[t]{2}{*}{ Risk-based $(n=635)$} & Yes & $57 \cdot 9(0 \cdot 37)$ & $50 \cdot 0(-1 \cdot 14)$ & $63 \cdot 3(2 \cdot 97)$ & $44 \cdot 2(-3 \cdot 21)$ \\
\hline & No & $42 \cdot 1(-0 \cdot 37)$ & $50 \cdot 0(1 \cdot 14)$ & $36 \cdot 7(-2 \cdot 97)$ & $55 \cdot 8(3 \cdot 21)$ \\
\hline \multirow[t]{2}{*}{ Multi-hazard $(n=571)$} & Yes & $18 \cdot 7(-5 \cdot 37)$ & $35 \cdot 1(-0 \cdot 22)$ & $59 \cdot 5(9 \cdot 83)$ & $12 \cdot 8(-5 \cdot 95)$ \\
\hline & No & $81 \cdot 3(5 \cdot 37)$ & $64.9(0 \cdot 22)$ & $40 \cdot 5(-9 \cdot 83)$ & $87 \cdot 2(5 \cdot 95)$ \\
\hline
\end{tabular}

* Adjusted residuals greater than $\pm 1 \cdot 96$ are highlighted in bold as they are more extreme than what would be expected if the null hypothesis of independence was true.

$\dagger$ The difference in the number of components compared to the total number indicated in the heading comprises missing or unknown observations.

introduction. For the risk factor period, both targeted (e.g. sampling at the end of high risk period to demonstrate freedom from disease) and stratified risk-based approaches (varying sampling intensities between seasons) were reported.

\section{DISCUSSION}

To the authors' knowledge, this is the first systematic analysis of publicly and privately funded AHS components in EU Member States and Switzerland. Generating an overview of all active AHS components covering the public and private sectors and the full range of hazards and species was challenging as existence and design of surveillance components are generally not systematically documented [31]. Hence, gaps and opportunities were identified not just related to AHS design, but also to the ease and quality of data collection. This survey showed that the public and private sectors applied a range of activities at the national and regional level in addition to obligatory
EU requirements. Even though data quality did not allow any in-depth between-country comparisons, observed patterns across the whole dataset are considered to provide valuable insights into how AHS was performed in 2011 in the ten study countries included in the analysis.

\section{Differences between partner and non-partner countries}

Systematic differences may exist between partner and non-partner countries due to the following reasons: partner countries were comprised only of EU-12 Member States predominantly situated in Central and Western Europe, who have a strong interest in animal health surveillance, which led to their participation in the RISKSUR project. This selection bias was aimed to be reduced by also incorporating EU-15 (CZ) and EU-25 (BG) Member States in the group of non-partner countries, which, however, also included three EU-12 Member States (DK, IE, IT) thus comprising a more heterogeneous group of 
Table 5. Percentage of risk-based sampling components $(n=366)$ stratified by risk factor $(95 \%$ confidence interval). Multiple selections of risk factors per components were allowed. The risk-based components were a subset of active surveillance components $(n=664)$ recorded by 10 study countries for the year 2011

\begin{tabular}{|c|c|c|c|}
\hline Risk factor & $\begin{array}{l}\text { Percentage of } \\
\text { components }(95 \% \mathrm{CI})\end{array}$ & Main type & $\begin{array}{l}\text { Comments (remaining components either } \\
\text { other type or unknown) }\end{array}$ \\
\hline Production type & $45 \cdot 1 \%(39 \cdot 9-50.3)$ & Targeted & $\begin{array}{l}\text { 142/165: Only breeding animals; } 96 / 142 \text { : } \\
\text { Surveillance in artificial insemination } \\
\text { centres }\end{array}$ \\
\hline Age & $24 \cdot 3 \%(20.0-29 \cdot 0)$ & Targeted & $\begin{array}{l}\text { 36/89: Serological components targeting } \\
\text { only older animals; } 37 / 89: \text { BSE/scrapie } \\
\text { components targeting older animals } \\
\text { only }\end{array}$ \\
\hline Region & $13 \cdot 1 \%(9 \cdot 8-17 \cdot 0)$ & Stratified & $\begin{array}{l}\text { 27/48: Surveillance intensity depended on } \\
\text { epidemiological situation in the region }\end{array}$ \\
\hline Herd size & $11 \cdot 2 \%(8 \cdot 2-14 \cdot 9)$ & Targeted & $\begin{array}{l}\text { 37/41: Only large sized farms were } \\
\text { targeted }\end{array}$ \\
\hline Time period & $6 \cdot 0 \%(3 \cdot 8-9 \cdot 0)$ & Varies & $\begin{array}{l}\text { Restricted to high-risk period (e.g. } \\
\text { demonstrate freedom at end of high-risk } \\
\text { period) or different sampling intensity } \\
\text { between seasons }\end{array}$ \\
\hline Production for human consumption & $3 \cdot 6 \%(1 \cdot 9-6 \cdot 0)$ & Targeted & \\
\hline Farm factors & $3 \cdot 8 \%(2 \cdot 1-6 \cdot 3)$ & Varies & 5/13: Targeting only outdoor farms \\
\hline Species & $2 \cdot 5 \%(1 \cdot 1-4 \cdot 6)$ & Varies & $\begin{array}{l}\text { Since components were generally split by } \\
\text { species, this risk factor was } \\
\text { predominantly recorded for avian } \\
\text { influenza (distinction between wild bird } \\
\text { and waterfowl species) }\end{array}$ \\
\hline Event & $2 \cdot 2 \%(0.9-4 \cdot 3)$ & Targeted & Testing prior to transport \\
\hline Disease status of the herd & $2 \cdot 2 \%(0.9-4 \cdot 3)$ & Targeted & E.g. certified free herds $v s$. non-free herds \\
\hline Trade & $1 \cdot 1 \%(0.3-2 \cdot 8)$ & Varies & $\begin{array}{l}\text { Trade volume (e.g. out-degree } \\
\text { movements) }\end{array}$ \\
\hline Previous & $2 \cdot 2 \%(0.9-4 \cdot 3)$ & Stratified & Previous irregularities or positive findings \\
\hline Various & $0.8 \%(0.2-2 \cdot 4)$ & n.a. & \\
\hline Unknown & $1 \cdot 9 \%(0.8-3 \cdot 9)$ & n.a. & \\
\hline
\end{tabular}

n.a., Not applicable.

Central, Southern and Southeastern European countries. Furthermore, potential differences in the efforts of collecting the data, data accessibility and availability of contacts may have occurred given that partner countries may have had better contacts to relevant institutions and a stronger interest in data collection given their participation in the project consortium. Acknowledging these potential differences between partner and non-partner countries, we stratified descriptive results and tested for the effect of partner country (yes/no) as part of the bivariate analyses.

Descriptive results indicated significant differences between partner and non-partner countries for the variables management (category 'both'), legal obligation (EU, private and none) and risk-based sampling (yes/no). The differences in management and legal obligation may indicate stronger investment of partner countries in non-EU regulated privately managed surveillance activities compared to non-partner countries. Another explanation may be that private and voluntary components were better captured in partner than non-partner countries given that data collection was highly challenging, so that a variety of information sources had to be screened and over 20 contacts were approached in some countries as part of data collection [34]. The difference in the percentage of riskbased components was not as pronounced as for the variables legal obligation and management. Avian influenza virus and Aujeszky's disease virus were the only hazards for which the total number of components justified a comparison at the hazard level between partner and non-partner countries. For avian influenza, the difference was driven by one nonpartner country recording eight surveillance 
components, all of which were risk-based as opposed to one to four components recorded by other countries, some of which were risk-based, while others were not. For Aujeszky's disease virus, wildlife components were only recorded in partner countries $(n=$ $2)$, none of which were risk-based, and abattoir surveillance was recorded as risk-based in both nonpartner countries but only in one out of four partner countries recording this component. Therefore, we conclude that the difference can be considered as spurious given that the percentages are to some degree affected by the number of recorded components.

Bivariate results indicated a significant effect of partner versus non-partner countries for the variables management (category 'both') and objective (category 'prevalence estimation'), but not for the four compared livestock species groups. For the variable management, the same arguments apply as discussed above. The higher number of components aimed at prevalence estimation may indicate that partner countries invest relatively more to assess changes in the hazard situation (e.g. antimicrobial resistance).

\section{Surveillance objective and means of data collection}

For active surveillance components, demonstrating freedom from disease was the most common objective, while early detection was least common. These objectives are in fact closely linked as activities to demonstrate freedom from disease are generally based on annual surveys, after which early detection is needed to maintain confidence in freedom until the next survey. Early detection activities were underrepresented in the data as passive surveillance components were excluded a priori given that they were considered too similar between countries. However, for many hazards passive surveillance is the predominant early detection component as it is continuously performed on a daily basis across the entire domestic (and wildlife) animal population. Furthermore, it can be highly cost-effective as testing is only performed if disease is suspected. For example Welby et al. [35] estimated detection probability (component sensitivity; CSe) and cost-effectiveness (CSe/cost in $€ 1000$ ) for three surveillance components targeting bluetongue virus serotype 8 in Belgium and The Netherlands. Based on a within-herd prevalence of $20 \%$ and the assumption that disease awareness is high, passive surveillance resulted in the highest probability of detection $(\mathrm{CSe}=0.99)$ compared to active cross-sectional surveys (CSe: $0 \cdot 73-0 \cdot 75)$ and sentinel surveillance
(CSe: $0 \cdot 29-0 \cdot 33)$ and a cost-efficiency ratio of 1.38 compared to 0.52 (survey) and 0.41 (sentinel). However, sensitivity of passive surveillance is influenced by the clinical effects of disease, rate of transmission, population structure (e.g. herd size, production system), disease awareness of animal owners and veterinarians, and their preparedness to report $[35,36]$. Therefore, it is important to also evaluate the sensitivity of passive surveillance [36], compare strategies to enhance disease awareness (e.g. awareness campaigns, training, adequacy of compensation) and reporting, and assess the acceptability by key stakeholders [37-39]. Effective dissemination of surveillance results to farmers and the public can also enhance engagement of these stakeholders and improve participation.

Given these limitations, active surveillance and syndromic surveillance may effectively enhance early detection [29]. Syndromic surveillance is also a (near) real-time surveillance activity aimed at early detection. Compared to passive surveillance, syndromic surveillance is less observer-dependent (depending on data source) and may detect abnormalities (e.g. in animal performance) before clinical signs occur, thus potentially resulting in enhanced timeliness. As part of One Health, syndromic surveillance in animal populations also provides opportunities for the early detection of public health risks [40]. Welby et al. [35] estimated the probability of syndromic surveillance to detect bluetongue disease virus serotype 8 in the Netherlands as 0.98 and 0.99 for milk production data assuming a within-herd prevalence of $2 \%$ and $20 \%$, respectively. Despite the high effectiveness that can be achieved via syndromic surveillance, its application requires access to data sources that are sensitive to changes in the level of disease in the population [40] and efficient algorithms that can detect potential outbreak signals [41]. The current study identified 19 syndromic surveillance components recorded by four of the ten study countries. Ten of these components targeted multiple diseases, e.g. emerging diseases $(n=9)$ or many diseases $(n=1)$, whereas the remaining components were hazardspecific $(n=5)$ or targeted disease syndromes, i.e. mastitis or metabolic disorders $(n=4)$. Diagnostic material and pathology examinations were mentioned as predominant data sources $(n=9)$. Furthermore, production data $(n=5)$, information from practitioners $(n=1)$ and information sources of government, public and charity organizations $(n=1)$ were recorded, while the remaining three components 
included insufficient information on the data source. These data suggest that syndromic surveillance is not being fully utilized by all countries yet, which is also in line with Dorea et al. [40].

\section{Risk-based sampling and multi-hazard surveillance}

For active components aimed at early detection, comprehensive coverage is generally not cost-effective as disease needs to be detected at very low prevalence to fulfil the aim of early detection [42]. Therefore, riskbased and alternative approaches (e.g. syndromic surveillance) are important to consider to enhance the likelihood of (early) detection. Efforts to demonstrate freedom from disease and detect cases also benefit from risk-based approaches, especially risk-based sampling and risk-based requirement (i.e. incorporation of historical data) $[10,43]$, as the aim is to detect disease rather than providing representative estimates such as for prevalence estimation. The current data indicate that $50.0 \%, 63.3 \%$, and $57.9 \%$ of components with the objectives early detection, demonstrating freedom from disease, and case detection, respectively, included risk-based approaches. Risk-based strategies and multi-hazard surveillance were more commonly recorded for the private sector compared to the public sector. Since two thirds of private components were based on EU regulations, this may only be partly explained by less stringent legal requirements. Differences between countries in the extent components are managed publicly or privately may contribute to the higher likelihood of risk-based approaches in the private sector with some countries being more progressive than others. But the private sector may also be under higher pressure to demonstrate costeffectiveness, which is supported by the fact that the private sector more commonly focussed on aspects with higher economic importance (e.g. cattle and pigs, demonstrating disease freedom to ensure trade). Based on these findings it is hypothesized that riskbased sampling and multi-hazard surveillance could be incorporated more strongly as part of public animal health surveillance.

Albeit risk-based sampling was recorded for more than half of components, most of these risk-based components were targeted, only focussing on one population stratum instead of adjusting surveillance intensity according to the risk level of different strata. While it may be justified in some cases to only focus on a single stratum (e.g. serological surveillance in older animals), no statement can be made on the population strata not included. Therefore targeted risk-based sampling as recorded for most of the riskbased components is in fact similar to conventional designs, just being focussed on a single population stratum. Stratified risk-based sampling in contrast distinguishes between high- and low-risk strata of the population. Since all animals have a nonzero probability of being selected, probabilistic statements can still be made for the entire population, but surveillance sensitivity can be increased given a constant sample size or sample size can be reduced at constant target sensitivity. Furthermore, stratified risk-based sampling allows maintaining low level surveillance in low-risk strata to help retain awareness and provide incentives to comply with regulations, e.g. the withdrawal period for antibiotics [27].

Movement data provide opportunities to distinguish between farms having a high risk of introduction or spread based on the frequency of in- and out-degree movements and the number and characteristics of trading partners. However, animal movements were only recorded as a risk criterion by two countries (four components), one of which only targeted high turnover premises (i.e. targeted risk-based). Hence, opportunities exist to enhance utilization of quantitative (e.g. movement data), but also qualitative data (e.g. biosecurity level) to distinguish between risk levels of different population strata. The data provided little evidence of the application of alternative approaches such as participatory [44] or event-based surveillance [45].

\section{Legal requirements}

Many EU and national legislations still prescribe what has to be done (input-based) rather than what has to be achieved (output-based) and generally focus on a single pathogen. Such input-based requirements do not provide much stimulus to evaluate alternative surveillance designs in order to identify the most cost-effective approach for the specific population to be targeted. Even though input-based standards are simple to compare between countries, they may result in low sensitivity in some population strata and excessive sample numbers in others [9]. EU countries vary considerably in their population structure, trading patterns, hazard situation, and risk factors. Output-based standards allow tailoring surveillance to the population of interest. However, epidemiologically sound application of output-based standards requires epidemiological expertise, knowledge regarding the target population, good data quality, and transparency. Interestingly, more 
recent regulations (e.g. 2006/88/EC for aquatic animals, 2007/268/EC for avian influenza virus, 1266/2007 for bluetongue disease virus - see Supplementary Table S2) encourage application of risk-based approaches and multi-hazard surveillance, indicating that the propagation of these alternative strategies in literature has informed legal requirements. Also, the new EU Animal Health Law explicitly emphasizes the need to take into account the epidemiology of disease, risk factors and characteristics of the target population (Article 27) and allows application of alternative strategies such as accounting for historical data to maintain disease free status [Article 36(1d)]. Hence, the new EU Animal Health Law provides enhanced opportunities to apply alternative surveillance approaches to increase effectiveness and cost-efficiency of surveillance.

\section{Importance of hazards}

Economic importance and zoonotic potential (7/10 of the most frequent hazards have zoonotic potential) appear to be important drivers for surveillance. As a result, species with little economic value such as pets and wildlife were less frequently covered, which presents another gap in surveillance. For zoonotic diseases, many alternative approaches have been promoted under the concept of One Health including systems thinking, participatory approaches and priority setting [46-49], which can only be achieved via effective collaboration between sectors (publicprivate) and disciplines (animal-public health). Transparent documentation of surveillance components and formal assessments of the entire surveillance system for a given hazard may also provide opportunities to inform or benefit from these approaches and thus possibly enhance convergence between human and veterinary health agencies. Such One Health approaches are increasingly promoted and present true opportunities to advance the effectiveness of zoonotic disease surveillance, early preparedness and protection of human and animal health in the EU.

\section{Sources of bias}

Selection bias might have occurred by including all partner countries and selecting five non-partner countries. However, a systematic and transparent selection process with clearly specified criteria was applied in an effort to minimize selection bias. Due to time and resource constraints, data collection could not be expanded to all EU Member States.
Furthermore, information bias may have occurred due to differences between countries in the availability of information and efforts made to seek additional information, centralisation of efforts, and willingness of contacts to share information. The efforts required collecting information on existing public and private surveillance activities covering all hazards and animal species exceeded expectations by far. Therefore, various grey literature sources had to be screened and contact persons approached to gather information on the full range of activities. Many countries have no centralised system capturing at least the existence and design of surveillance activities. Furthermore, surveillance documentation was more difficult to obtain for the private than the public sector. In a post-hoc questionnaire, data collectors ranked statements that $(a)$ the existence and $(b)$ the design of surveillance was adequately documented in their country, resulting in a median rank of 4 and 3 out of 5 (range 2-5) for public and 2 for both out of 5 (range 1-4) for private components [34]. Data collection also indicated considerable differences between countries regarding evaluation and dissemination of surveillance efforts as well as collaborations between the public and private sector. Furthermore, while some countries have a strongly centralized system (e.g. NL, SE, UK), others are strongly decentralized (e.g. DE, ES, FR). Regional variability is more pronounced in decentralized countries, which is difficult to capture. Last, some countries were concerned about confidentiality, so that participation was only agreed on if data were presented in aggregated form and countries were anonymized. These challenges were reflected by a wide range in the number of reported components and percentage of obligatory EU components covered in the countries' datasets. This latter percentage is underestimated as it is sometimes affected by the way components were split (i.e. a country merging components not merged by other countries). However, completeness has affected data quality in some countries, so that results have to be interpreted with care and no between-country comparisons were attempted.

Another major limitation related to differences in applying terminology to specific components, which led to initial high variability in how variables were categorized between countries. As a result, extensive consistency checks were carried out and a terminology working group established to improve the comparability of data. These inconsistencies in applying terminology and general inconsistencies between data collected, as shown in this study, could therefore be used as a basis to argue for a standardized documentation of the design of surveillance activities within the EU. Documentation guidelines or 
standard forms have for example been developed by EFSA for surveillance of Echinococcus multilocularis [50] and by the EU reference laboratory for surveillance of fish diseases (http://www.eurl-fish.eu/Activities/ survey_and_diagnosis.aspx). But no standard requirements have been agreed on, which provide sufficient information to create an informed overview, but sufficient flexibility to accommodate different approaches and preserve the necessary degree of confidentiality.

\section{Recommendations regarding surveillance design}

It is difficult to make general recommendations regarding surveillance design as it depends on many factors such as the hazard and population under surveillance, the hazard situation and objective. When judging surveillance design, it is important to consider the surveillance system as a whole including its passive and active components as components may complement each other. This systems approach allows $(a)$ identification of surveillance gaps (i.e. sectors or age groups not covered) and components with suboptimal effectiveness and (b) assessment of opportunities to increase effectiveness and economic efficiency.

The effectiveness of surveillance approaches can only be optimized if various surveillance designs are formally compared as demonstrated manifold in published literature over recent years. This may include comparisons of different testing regimens, sampling points and risk strata, strategies to enhance awareness or incorporation of historical data. Hence, systematic documentation of current designs, evaluation and comparisons with alternative strategies provide opportunities to select more effective and efficient surveillance approaches. However, improved transparency and documentation of design details may be needed to allow application of more flexible approaches.

\section{Recommendations derived from the process of collecting the data}

If registration of surveillance activities at country level was centralized, this would provide an overview and allow for better coordination of efforts to be made between the public and private sector. Furthermore, a minimum set of design variables could be defined in advance in order to achieve consistency in documentation. This could be achieved through the use of the surveillance design framework developed by the RISKSUR project, which provides a tool that can be used for standardized documentation of surveillance efforts (http://webtools.fp7-risksur.eu/).
Moreover, despite a common source of definitions, terminology may lead to differences in interpretations when applied to specific components, as shown by this study. Therefore, illustrating the correct application of terminology based on specific components may be useful to supplement definitions and thus achieve better consistency (http://www.fp7-risksur.eu/terminology). Given that surveillance can be perceived to be a sensitive topic, it is also important to clearly explain to contributing parties the purpose and expected outcome of more transparent documentation. Confidentiality concerns may limit the application of alternative strategies, as these require transparent documentation to assess the adequacy of assumptions and design specifications. However, these limitations stand in contrast to the claim that surveillance is a public good [51]. Hence, there is a joint responsibility to address factors limiting transparent documentation of surveillance activities.

All these aspects do not just pertain to this study but may cause problems with any compilation of surveillance activities across EU Member States (e.g. EFSA reports, EU summary reports). Hence, these gaps hinder information sharing in general and limit the ability to integrate information from various sources (e.g. active/passive, public/private, different species) in a meaningful manner. It is hypothesized that increased transparency would benefit all parties by enhancing trust, facilitating meaningful comparisons, and allowing more targeted complementation of activities based on the evaluation of the entire surveillance system rather than its individual components in isolation.

\section{CONCLUSIONS}

The study identified several gaps (lack of systematic documentation, inconsistent application of terminology, little evidence of surveillance in species with low economic importance) and opportunities (e.g. better uptake of alternative methods, increased use of stratified risk-based sampling, application of novel approaches promoted as part of One Health). The greater flexibility provided by the new EU Animal Health Law means that systematic evaluation of surveillance alternatives will be required to ensure that surveillance is as efficient and effective as possible [52].

\section{SUPPLEMENTARY MATERIAL}

For supplementary material accompanying this paper visit https://doi.org/10.1017/S0950268816002697. 


\section{ACKNOWLEDGEMENTS}

This project has received funding from the European Union's Seventh Framework Programme (FP7/20072013) under grant agreement no. 310806. The authors acknowledge all data collectors, particularly Jenny Frössling (SVA), Sophie Molia (CIRAD), Iveta Karabozhilova (RVC), Jo Hardstaff (RVC), Detlef Höreth-Böntgen (FLI), Angel Ortiz-Pelaez (APHA), Mark Rigby (AHPA), Thomas Rosendal (SVA), Helene Wahlström (SVA). We also acknowledge Till Ittermann for advice on statistical analysis and thank the three referees for their valuable comments on the manuscript.

\section{DECLARATION OF INTEREST}

None.

\section{REFERENCES}

1. Anonymous. Impact assessment accompanying the document 'Proposal for a regulation of the European Parlament and of the Council on animal health'. In SWD(2013) 161 final, Csw document, 2013. Brussels, p. 183 (http://ec.europa.eu/food/animals/docs/ ah-law-impact-assesment_en.pdf).

2. Hoinville LJ, et al. Proposed terms and concepts for describing and evaluating animal-health surveillance systems. Preventive Veterinary Medicine 2013; 112: 1-12.

3. Haesler B, Howe KS, Staerk KDC. Conceptualising the technical relationship of animal disease surveillance to intervention and mitigation as a basis for economic analysis. Bmc Health Services Research 2011; 11.

4. Hoinville LJ, et al. Animal health surveillance terminology. In Final Report from Pre-ICAHS Workshop, 2013 (http:// www.defra.gov.uk/ahvla-en/files/icahs-workshop-report. pdf).

5. European Communities. A new animal health strategy for the European Union (2007-2013) where 'Prevention is better than cure'. In $C O M(2007)$ final. 2007. Office for Official Publications of the European Community: Luxembourg (http://www.oie.int/fileadmin/Home/eng/ Support_to_OIE_Members/docs/pdf/EU_Animal_Health_ Strategy_EN_.pdf).

6. Van Asselt ED, et al. Overview of available methods for risk based control within the European Union. Trends in Food Science \& Technology 2012; 23: 51-58.

7. European Union. Regulation (EU) 2016/429 of the European Parliament and of the Council on transmissible animal diseases and amending and repealing certain acts in the area of animal health ('Animal Health Law'), 2016 (http://eur-lex.europa.eu/legal-content/EN/ TXT/PDF/?uri=OJ:L:2016:084:FULL\&from=EN).

8. Cameron AR. The consequences of risk-based surveillance: Developing output-based standards for surveillance to demonstrate freedom from disease. Preventive Veterinary Medicine 2012; 105: 280-286.

9. De Massis F, Petrini A, Giovannini A. Reliability evaluation of sampling plan fixed by Council Directive 91/ 68/EEC for the maintenance of officially brucellosis-free flock status. Journal of Veterinary Medicine. B, Infectious Diseases and Veterinary Public Health 2005; 52: 284-290.

10. Alban L, et al. Towards a risk-based surveillance for Trichinella spp. in Danish pig production. Preventive Veterinary Medicine 2008; 87: 340-357.

11. Martin PAJ. Current value of historical and ongoing surveillance for disease freedom: surveillance for bovine Johne's disease in Western Australia. Preventive Veterinary Medicine 2008; 84: 291-309.

12. Greiner M, Dekker A. On the surveillance for animal diseases in small herds. Preventive Veterinary Medicine 2005; 70: 223-234.

13. Reist M, Jemmi T, Stärk KDC. Policy-driven development of cost-effective, risk-based surveillance strategies. Preventive Veterinary Medicine 2012; 105: 176-484.

14. Alban L, et al. Towards a standardised surveillance for Trichinella in the European Union. Preventive Veterinary Medicine 2011; 99: 148-160.

15. Schuppers ME, et al. Implementing a probabilistic definition of freedom from infection to facilitate trade of livestock: putting theory into praxis for the example of bovine herpes virus-1. Preventive Veterinary Medicine 2012; 105: 195-201.

16. Alba A, et al. Assessment of different surveillance systems for avian influenza in commercial poultry in Catalonia (North-Eastern Spain). Preventive Veterinary Medicine 2010; 97: 107-118.

17. Böhning D, Greiner M. Modelling cumulative evidence for freedom from disease with applications to BSE surveillance trials. Journal of Agricultural, Biological, and Environmental Statistics 2006; 11: 280-295.

18. Martinez M-J, et al. Methodological approach for substantiating disease freedom in a heterogeneous small population. Application to ovine scrapie, a disease with a strong genetic susceptibility. Preventive Veterinary Medicine 2010; 95: 108-114.

19. Foddai A, et al. Comparison of output-based approaches used to substantiate bovine tuberculosis free status in Danish cattle herds. Preventive Veterinary Medicine 2015; 121: 21-29.

20. More SJ, et al. Defining output-based standards to achieve and maintain tuberculosis freedom in farmed deer, with reference to member states of the European Union. Preventive Veterinary Medicine 2009; 90: 254-267.

21. Riviere J, et al. Sensitivity of bovine tuberculosis surveillance in wildlife in France: a scenario tree approach. PloS ONE 2015; 10.

22. Wahlström $\mathbf{H}$, et al. Demonstrating freedom from Mycobacterium bovis infection in Swedish farmed deer using non-survey data sources. Preventive Veterinary Medicine 2010; 94: 108-118.

23. Frössling $\mathbf{J}$, et al. Surveillance system sensitivities and probability of freedom from Mycobacterium avium subsp. paratuberculosis infection in Swedish cattle. Preventive Veterinary Medicine 2013; 108: 47-62. 
24. More SJ, et al. The effect of alternative testing strategies and bio-exclusion practices on Johne's disease risk in test-negative herds. Journal of Dairy Science 2013; 96: 1581-1590.

25. Martin PAJ, et al. Demonstrating freedom from disease using multiple complex data sources 2: Case studyclassical swine fever in Denmark. Preventive Veterinary Medicine 2007; 79: 98-115.

26. Welby $\mathbf{S}$, et al. Bluetongue surveillance system in Belgium: a stochastic evaluation of its risk-based approach effectiveness. Preventive Veterinary Medicine 2013; 112: 48-57.

27. Alban L, et al. Comparison of risk-based versus random sampling in the monitoring of antimicrobial residues in Danish finishing pigs. Preventive Veterinary Medicine 2016; 128: 87-94.

28. Riviere $\mathbf{J}$, et al. Bovine tuberculosis surveillance in cattle and free-ranging wildlife in EU Member States in 2013: a survey-based review. Veterinary Microbiology 2014; 173: 323-331.

29. Rodriguez-Prieto V, et al. Systematic review of surveillance systems and methods for early detection of exotic, new and re-emerging diseases in animal populations. Epidemiology and Infection 2015; 143: 2018-2042.

30. Binder S, et al. Emerging infectious diseases: Public health issues for the 21st century. Science 1999; 284: 1311-1313.

31. Schauer B, et al. 'Surveillance is a public good'-but how public is it? in EPIZONE 8th Annual Meeting, 2014. Copenhagen, Denmark (http://www.fp7-risksur.eu/ sites/default/files/documents/publications/EpiZone2014_ BSchauer_HowPublicIsSurveillance.pdf).

32. Anonymous. Terminology: frequently asked questions, 2015 (http://www.fp7-risksur.eu/terminology/faq).

33. Sharpe D. Your chi-square test is statistically significant: now what? In Practical Assessment, Research \& Evaluation, 2015, p. 1-10 (http://pareonline.net/getvn.asp? $\mathrm{v}=20 \& \mathrm{n}=8$ ).

34. Schauer B, et al. Data collection protocols and guidelines. In Deliverable No. 1·5, 2013, p. 33 (http://www. fp7-risksur.eu/sites/default/files/documents/Deliverables/ RISKSUR $\% 20 \% 28310806 \% 29 \% 20 \mathrm{D} 1 \cdot 5$.pdf).

35. Welby S, et al. Effectiveness and cost efficiency of different surveillance components for proving freedom and early detection of disease: bluetongue serotype 8 in cattle as case study for Belgium, France and the Netherlands. Transboundary and Emerging Diseases. doi:10.1111/ tbed.12564. Published online: 26 September 2016.

36. Hadorn DC, Haracic SS, Stärk KDC. Comparative assessment of passive surveillance in disease-free and endemic situation: example of Brucella melitensis surveillance in Switzerland and in Bosnia and Herzegovina. BMC Veterinary Research 2008; 4: 1-9.

37. Schulz K, et al. Hunters' acceptability of the surveillance system and alternative surveillance strategies for classical swine fever in wild boar-a participatory approach. BMC Veterinary Research 2016; 12.

38. Calba C, et al. The added-value of using participatory approaches to assess the acceptability of surveillance systems: the case of bovine tuberculosis in Belgium. $P L O S O N E$ 2016; 11.

39. Brugere C, Onuigbo DM, Morgan KL. People matter in animal disease surveillance: challenges and opportunities for the aquaculture sector. Aquaculture. doi:http:// dx.doi.org/10·1016/j.aquaculture.2016·04·012. Published online: 16 April 2016.

40. Dorea FC, Sanchez J, Revie CW. Veterinary syndromic surveillance: current initiatives and potential for development. Preventive Veterinary Medicine 2011; 101: 1-17.

41. Dorea FC, et al. Syndromic surveillance using veterinary laboratory data: algorithm combination and customization of alerts. PLOS ONE 2013; 8 .

42. Cameron A, Manual of basic animal disease surveillance, 2012. African Union: Interafrican Bureau for Animal Resources: Nairobi, Kenya (http://www. au-ibar.org/component/jdownloads/finish/76/1546).

43. Schwermer H, Reding I, Hadorn DC. Risk-based sample size calculation for consecutive surveys to document freedom from animal diseases. Preventive Veterinary Medicine 2009; 92: 366-372.

44. Paolotti D, et al. Web-based participatory surveillance of infectious diseases: the Influenzanet participatory surveillance experience. Clinical Microbiology and Infection 2014; 20: 17-21.

45. Gossner CM, et al., Event-based surveillance of foodand waterborne diseases in Europe: 'urgent inquiries' (outbreak alerts) during 2008 to 2013. Eurosurveillance. 2015; 19-28.

46. The World Bank. People, pathogens and our planet, 2010 (http://siteresources.worldbank.org/INTARD/Resources/ PPP_Web.pdf).

47. WHO. Taking a Participatory Approach to Development and Better Health: Examples from the Regions for Health Network. Gravesen N, ed. 2015 (http://www. euro.who.int/_data/assets/pdf_file/0007/294064/Takingparticipatory-approach-development-health-malmo-ska ne.pdf).

48. Stark KDC, et al. One Health surveillance - more than a buzz word? Preventive Veterinary Medicine 2015; 120: 124-130.

49. Binot A, et al. A framework to promote collective action within the One Health community of practice: using participatory modelling to enable interdisciplinary, cross-sectoral and multi-level integration. One Health 2015; 1: 44-48.

50. EFSA. Scientific and technical assistance on Echinococcus multilocularis infection in animals. EFSA Journal 2012, p. 2973. European Food Safety Authority: Parma, Italy.

51. FAO. Challenges of animal health information systems and surveillance for animal diseases and zoonoses. In Proceedings of the International Workshop organized by FAO, 2011. Rome, Italy (http://www.fao.org/3/ a-i2415e.pdf).

52. Staerk KDC, Haesler B. The value of information: current challenges in surveillance implementation. Preventive Veterinary Medicine 2015; 122: 229-234. 